\title{
Vigas de concreto reforçadas com bambu Dendrocalamus giganteus. II: Modelagem e critérios de dimensionamento
}

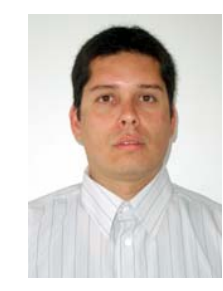

\author{
Humberto C. Lima Júnior ${ }^{1}$, Fábio L. Willrich ${ }^{1} \&$ Gilmar Fabro² \\ ${ }^{1}$ UNIOESTE. Rua Universitária 2069, CEP 85814-110, Cascavel, PR. Fone: (45) 220-3221. E-mail: correialima@unioeste.br \\ (Foto); flwillrich@unioeste.br \\ 2 ITAIPU Binacional. Av. Tancredo Neves 6731, CP 1555, CEP 85856-000, Foz do Iguaçu, PR. Fone: (45) 520-6220. \\ E-mail: fabro@itaipu.gov.br
}

Protocolo $60-6 / 5 / 2004$ - Aprovado em 6/4/2005

\begin{abstract}
Resumo: Este trabalho corresponde à segunda parte de uma publicação sobre o comportamento estrutural de vigas de concreto reforçadas com bambu, na qual se apresenta e discute a modelagem dessas estruturas para, em seguida, serem apresentadas sugestões e hipóteses para o dimensionamento desses elementos estruturais. Para tanto, utilizou-se um modelo computacional baseado no Método dos Elementos Finitos, ao qual foram incorporadas sub-rotinas com as leis constitutivas do bambu e do concreto. Para calibração do modelo lançou-se mão dos dados experimentais de oito vigas de concreto reforçadas com bambu. Os resultados obtidos com o modelo computacional foram comparados com os experimentais, observando-se grande concordância. Finalmente, sugerem-se critérios de dimensionamento, os quais foram aplicados em um exemplo prático.
\end{abstract}

Palavras-chave: reforço não metálico, elemento estrutural, material alternativo, software

\section{Concrete beams reinforced with Dendrocalamus giganteus bamboo. II: Modeling and design criterions}

\begin{abstract}
This paper corresponds to the second part of a publication concerning the structural behaviour of concrete beams reinforced with bamboo. Modelling of concrete beams reinforced with bamboo-splint are presented and discussed. In addition, some design suggestions and hypotheses are presented. To perform the study, a Finite Element Program was used and some procedures were programmed and linked to it. The program was calibrated with the experimental data of eight concrete beams reinforced with bamboo-splint, whose results presented great accuracy. Finally, some design procedures were suggested and a practical example is given.
\end{abstract}

Key words: non-metallic reinforcement, structural element, alternative material, software

\section{INTRODUÇÃO}

A possibilidade de utilização do bambu como reforço no concreto vem sendo estudada por diversos pesquisadores, desde o início do século passado. Desde então, diversas pesquisas vêm sendo desenvolvidas; todavia, poucas conclusões foram obtidas sobre o tema (Ghavami \& Hombeck, 1981; Beraldo, 1987; Ferrão \& Freire, 1995; Lima Jr. et al., 1996; Rosa, 2002; Ferreira et al., 2001). Com base nisso, observa-se que a falta de uma normalização adequada, critérios claros de dimensionamento e tecnologias convenientes, vêm limitando a utilização do bambu como reforço no concreto.
Outro ponto de relutância da utilização do bambu como reforço no concreto é a questão da durabilidade. Cordero (1990) relatou que muitas moradias construídas com bambu e madeira se degradam em dois ou três anos, quando não são utilizados os procedimentos corretos; entretanto, este material foi largamente utilizado como reforço de paredes de terra crua, apresentando durabilidade superior a 100 anos (Lopez, 1974).

Um dos primeiros estudos sobre a utilização do bambu, como reforço em vigas de concreto armado realizado no Brasil, foi desenvolvido por Culzoni (1985) que, em seu trabalho, estudou o comportamento de duas vigas de concreto reforçadas com bambu, comparando-as com uma viga armada com aço. Culzoni (1985) observou que as vigas de bambu apresentaram rigidez e 
capacidade de carga inferiores à de aço, porém se observa, neste trabalho, que tanto o dimensionamento das tiras de bambu como o das barras de aço não seguiram qualquer critério para uma posterior comparação; ainda se verifica que as três vigas apresentaram modos de colapso distintos, não sendo possível se obter muitas conclusões.

Lima Jr. et al. (1994) ensaiaram três vigas de concreto reforçadas com bambu, as quais apresentavam taxas de armadura e tratamentos impermeabilizantes distintos. Os autores relataram que a primeira viga apresentou colapso por cisalhamento com tração excessiva na biela tracionada; a segunda, colapso por flexão com tração excessiva no reforço do bambu e a última, colapso por flexão com ruptura por compressão excessiva do concreto. Deste modo, verifica-se que, devido à grande variabilidade das variáveis envolvidas, os autores pouco ou quase nada concluíram sobre o tema; apesar disso, eles reafirmaram a viabilidade técnica da utilização desse material como reforço de vigas de concreto em pequenas edificações rurais.

Recentemente, Ferreira et al. (2001) ensaiaram três vigas de bambu e as compararam com uma viga de referência reforçada com aço; elas apresentavam seção transversal de $15 \mathrm{~cm}$ x $20 \mathrm{~cm}$ e vão teórico de $1,80 \mathrm{~m}$. Todas as vigas de bambu apresentavam taxas de armadura e tratamentos superficiais distintos das varas de bambu. Os autores reafirmaram a viabilidade técnica do emprego desse material como reforço em vigas de concreto; contudo, não apresentaram critérios claros que pudessem ser empregados para o dimensionamento desses elementos estruturais.

Com base no apresentado, pode-se constatar a necessidade da proposição de critérios para o dimensionamento das vigas de concreto armadas com bambu e de uma futura normalização do tema. Dentro desse contexto o trabalho relata a segunda parte de um projeto de pesquisa através do qual se vem investigando o comportamento das vigas de concreto reforçadas com bambu. Na primeira parte, relatou-se o estudo experimental de oito vigas de bambu e, a partir deste, buscouse pesquisar a influência da taxa de armadura longitudinal e da relação área/perímetro das varas de bambu no comportamento desses elementos estruturais. Com base nesta primeira fase realizou-se um segundo estudo, visando à modelagem das vigas de concreto reforçadas com bambu, por meio do Método dos Elementos Finitos e, em seguida, à criação de critérios de dimensionamento.

\section{MATERIAL E MÉTODOS}

\section{Modelo computacional}

Para modelagem e análise das vigas de concreto reforçadas com bambu utilizou-se um programa computacional, com base no Método dos Elementos Finitos (Krüger, 1989; Campos, 1993). Este programa foi desenvolvido para analisar pórticos planos de concreto armado e protendido e considera as nãolinearidades físicas do material e as geométricas da estrutura. A teoria utilizada é a teoria de vigas de Bernoulli-Kirchhoff e o elemento finito utilizado o de viga, inicialmente descrito em um sistema natural de coordenadas e, depois, transformado para um sistema auxiliar de coordenadas, cujas coordenadas de deslocamentos são dispostas segundo as coordenadas globais do pórtico e permitem a descrição de deslocamentos de corpo rígido. Na Figura 1 são apresentados o elemento finito utilizado e os sistemas de coordenadas.
A.

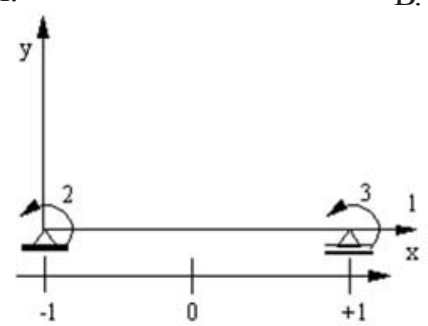

B.

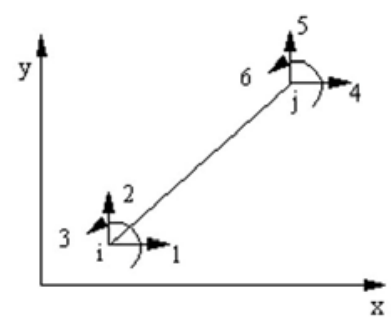

Figura 1. Elemento finito utilizado e sistemas de coordenadas: natural (A) e auxiliar (B)

Após a transformação para o sistema global de coordenadas, segue-se o método da rigidez direta. A resolução do sistema não-linear de equações, necessária para cada etapa do processo incremental, é realizada pelo método de Newton-Raphson, com controle de deslocamento. O programa consegue representar os fenômenos de "snap-through" e "snap-back" e, independentemente do método utilizado para a resolução do sistema não-linear de equações; ele segue o algoritmo de análise da estrutura numa etapa do carregamento, apresentada na Figura 2.

\section{Implementações}

Foram implementadas sub-rotinas em Linguagem Fortran, que continham as leis constitutivas do concreto, segundo o FIB (1999), e do bambu (Lima Jr. et al., 2000); em seguida, essas sub-rotinas foram acopladas ao programa ANAPROT (Krüger, 1989) no qual se realizaram pequenas modificações no código do programa original, para que este pudesse se comunicar com as sub-rotinas. O procedimento de integração numérica utilizado foi o mesmo para todas as sub-rotinas e será descrito a seguir.

Definidas as leis constitutivas dos materiais e a seção transversal do elemento estrutural de concreto armado, podese obter os esforços resistentes, utilizando-se um sistema local de coordenada $(\xi, \eta, \zeta)$, que passa pelo centro de gravidade da seção transversal, por meio da integração das tensões definidas para o concreto e para o bambu, em função das curvas tensãodeformação de cada material. Os esforços resistentes podem ser descritos com base nas Eqs. 1 e 2:

$$
\begin{gathered}
M_{\xi}=-\left(\int_{A_{c}} \sigma_{c}(\varepsilon) \cdot \eta \cdot d A+\sum_{n} A_{b_{n}} \cdot \sigma_{b}(\varepsilon)_{n} \cdot \eta_{n}\right) \\
N_{\xi}=-\left(\int_{A_{c}} \sigma_{c}(\varepsilon) \cdot d A+\sum_{n} A_{b_{n}} \cdot \sigma_{b}(\varepsilon)_{n}\right)
\end{gathered}
$$

Nas Eqs. 1 e $2, \sigma_{c}(\varepsilon)$ e $\sigma_{b}(\varepsilon)$ são as tensões no concreto e no bambu, respectivamente; $A_{c}$ é a área do concreto; $\eta$ é a ordenada de um ponto qualquer da seção no sistema de coordenada 


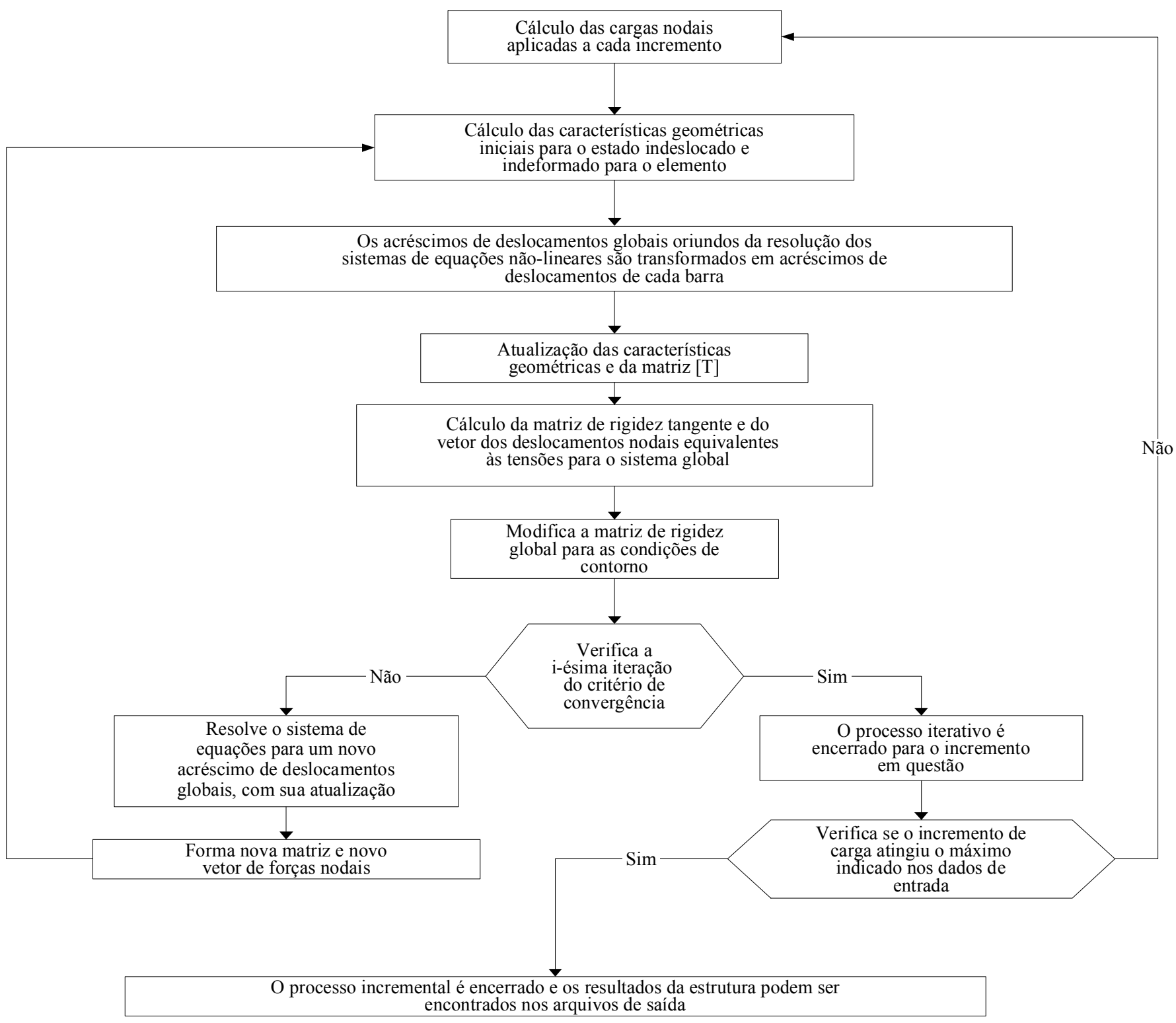

Figura 2. Algoritmo utilizado pelo programa para a análise de uma estrutura em uma etapa de carregamento

local; $A_{b n}$ e $\eta_{n}$ são a área e a ordenada da vara de bambu n, respectivamente. Na Figura 3 apresentam-se detalhes do sistema de coordenadas locais.

Analisando-se as Eqs.1 e 2, observa-se que os esforços resistentes contêm não só contribuição do bambu mas, também, a do concreto e, ainda, que para quantificar a parcela atribuída ao concreto, é conveniente a resolução das integrais presentes nessas equações. O processo numérico utilizado para resolução das integrais consiste em transformar as integrais de superfície sobre o domínio em integrais de linha ao longo do contorno, aplicando-se o teorema de Green no plano. Uma seção transversal qualquer é definida como uma poligonal fechada, percorrida no sentido anti-horário, atentando-se para o fato de que, em caso de aberturas internas, estas serão definidas por uma orientação horária. Na Figura 3 apresenta-se a definição do domínio de integração (Krüger, 1989).

Pela aplicação do teorema de Green no plano, a expressão de um termo genérico de um polinômio transformado para uma integral de contorno, é expressa pela Eq. 3:

$$
\int_{\mathrm{R}} \xi^{\mathrm{k}} \cdot \eta^{\mathrm{m}} \mathrm{dA}=\int_{\mathrm{C}} \frac{\xi^{\mathrm{k}+1} \cdot \eta^{\mathrm{m}}}{\mathrm{k}+1} \mathrm{~d} \eta=\sum_{\mathrm{j}=1}^{\mathrm{N}} \mathrm{G}_{\mathrm{k}, \mathrm{m}}
$$

na qual $\mathrm{N}$ representa o número de segmentos e $\mathrm{G}_{\mathrm{k}, \mathrm{m}}$ a integral de contorno do j-ésimo segmento da poligonal, defínido pelos vértices i e $\mathrm{i}+1$. As integrais $\mathrm{G}_{\mathrm{k}, \mathrm{m}}$ podem ser expressas pela Eq. 4:

$$
\mathrm{G}_{\mathrm{k}, \mathrm{m}}=\frac{1}{\mathrm{k}+1} \cdot \int_{\eta_{\mathrm{i}}}^{\eta_{\mathrm{i}+1} \xi^{\mathrm{k}+1}} \cdot \eta^{\mathrm{m}} \mathrm{d} \eta
$$

Considerando-se $\omega$ um incremento qualquer na ordenada $\eta$, pode-se escrever a Eq. 5:

$$
\begin{aligned}
& \eta=\eta_{i}+\omega \\
& \xi=\xi_{i}+\frac{\Delta \xi}{\Delta \eta} \cdot \omega
\end{aligned}
$$


A.

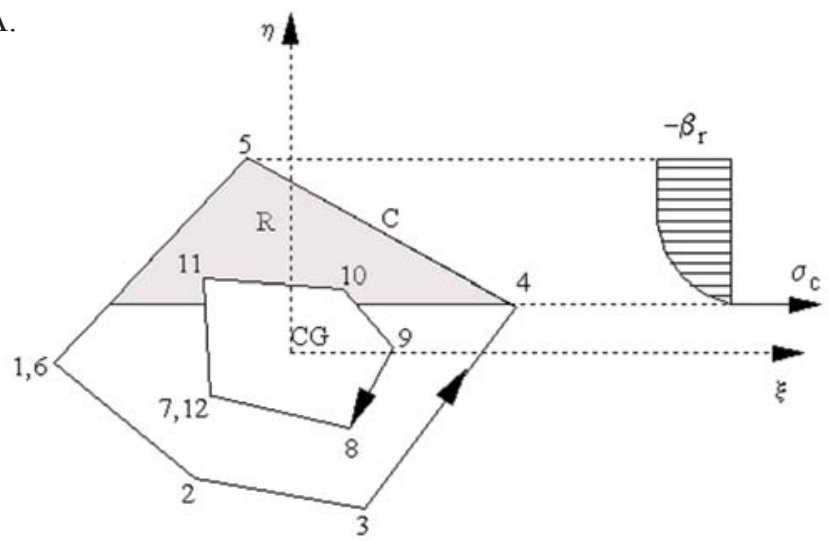

B.

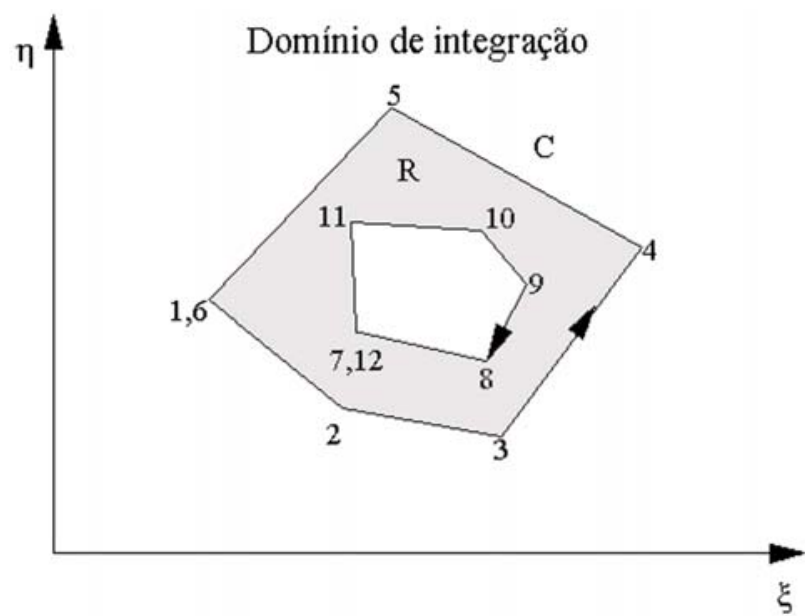

Figura 3. Detalhes do sistema de coordenada local (A) e domínio de integração da seção transversal analisada (B)

Substituindo a Eq. 5 na Eq. 4, obtém-se a equação Eq. 6:

$$
\mathrm{G}_{\mathrm{k}, \mathrm{m}}=\frac{1}{\mathrm{k}+1} \cdot \int_{0}^{\Delta \eta}\left(\xi_{\mathrm{i}}+\frac{\Delta \xi}{\Delta \eta} \cdot \omega\right)^{\mathrm{k}+1} \cdot\left(\eta_{\mathrm{i}}+\omega\right)^{\mathrm{m}} \mathrm{d} \omega
$$

na qual $\Delta \eta$ e $\Delta \xi$ são dados pela Eq. 7:

$$
\begin{aligned}
& \Delta \eta=\eta_{i+1}-\eta_{i} \\
& \Delta \xi=\xi_{i+1}-\xi_{i}
\end{aligned}
$$

Utilizando-se o procedimento anteriormente descrito, as equações Eq. 1 e 2 se tornam somatório de várias parcelas, funções de $\mathrm{G}_{\mathrm{k}, \mathrm{m}}$. Após o cálculo dessas funções segue-se o procedimento usual de utilização da integração numérica Gaussiana, para resolução das matrizes de rigidez do sistema.

O programa ANAPROT já disponibilizava as leis constitutivas para o concreto, segundo o CEB (1990), as quais foram adaptadas para o FIB (1999); assim, o valor da deformação $\varepsilon_{\text {co }}$, que é sugerido como -0,0022 pelo CEB (1990), foi substituído pelos valores sugeridos pelo FIB (1999), que podem ser calculados com base na Eq. 8:

$$
\varepsilon_{\mathrm{co}}=-0,0017-0,0010 \cdot\left(\frac{\mathrm{f}_{\mathrm{c}}}{\mathrm{f}_{\mathrm{cmo}}}\right)
$$

na qual $\mathrm{f}_{\mathrm{cmo}}$ é igual a $70 \mathrm{MPa}$ e $\mathrm{f}_{\mathrm{c}}$ é a resistência à compressão média do concreto. Ainda segundo o FIB (op. cit.), a tensão no concreto pode ser calculada com base na Eq.(9):

$$
\sigma_{\mathrm{c}}=\left[\frac{\left(\frac{\mathrm{E}_{\mathrm{c}}}{\mathrm{E}_{\mathrm{co}}}\right) \cdot\left(\frac{\varepsilon_{\mathrm{c}}}{\varepsilon_{\mathrm{co}}}\right)-\left(\frac{\varepsilon_{\mathrm{c}}}{\varepsilon_{\mathrm{co}}}\right)^{2}}{1+\left(\left(\frac{\mathrm{E}_{\mathrm{c}}}{\mathrm{E}_{\mathrm{co}}}\right)-2\right) \cdot\left(\frac{\varepsilon_{\mathrm{c}}}{\varepsilon_{\mathrm{co}}}\right)}\right] \cdot \mathrm{f}_{\mathrm{c}} \quad \varepsilon_{\mathrm{c}} \leq \varepsilon_{\mathrm{cu}}
$$

em que $\mathrm{E}_{\mathrm{co}}$ é o módulo de elasticidade secante do concreto, dado pela Eq. 10, $\mathrm{E}_{\mathrm{c}}$ é o módulo de elasticidade do concreto calculado pela Eq. 11 e $\varepsilon_{\mathrm{cu}}$ é a deformação última do concreto, dado pela Eq. 12:

$$
\begin{gathered}
\mathrm{E}_{\mathrm{co}}=\frac{\mathrm{f}_{\mathrm{c}}}{\varepsilon_{\mathrm{co}}} \quad(\mathrm{MPa}) \\
\mathrm{E}_{\mathrm{c}}=\alpha_{\mathrm{e}} \cdot \alpha_{\beta} \cdot\left[\mathrm{f}_{\mathrm{c}} / \mathrm{f}_{\mathrm{cmo}}\right]^{1 / 3} \quad(\mathrm{MPa}) \\
\frac{\varepsilon_{\mathrm{cu}}}{\varepsilon_{\mathrm{co}}}=\frac{1}{2} \cdot\left(\frac{1}{2} \cdot \frac{\mathrm{E}_{\mathrm{c}}}{\mathrm{E}_{\mathrm{co}}}+1\right)+\sqrt{\frac{1}{4} \cdot\left(\frac{1}{2} \cdot \frac{\mathrm{E}_{\mathrm{c}}}{\mathrm{E}_{\mathrm{co}}}+1\right)^{2}-\frac{1}{2}}
\end{gathered}
$$

sendo $\alpha_{\mathrm{e}}$ é igual a $21500 \mathrm{MPa}$ e $\alpha$ um coeficiente que depende do tipo de agregado graúdo que constitui o concreto, sendo para o agregado basáltico, igual a 1,2. As leis constitutivas do concreto submetido a tração, sugeridas pelo FIB (1999) podem ser expressas pela Eq. 13:

$$
\begin{gathered}
\sigma_{\mathrm{ct}}=\mathrm{E}_{\mathrm{c}} \cdot \varepsilon_{\mathrm{ct}} \quad \sigma_{\mathrm{ct}} \leq 0,9 \cdot \mathrm{f}_{\mathrm{ctm}} \\
\sigma_{\mathrm{ct}}=\mathrm{f}_{\mathrm{ctm}}-\frac{0,1 \cdot \mathrm{f}_{\mathrm{ctm}}}{0,00015-\frac{0,9 \cdot \mathrm{f}_{\mathrm{ctm}}}{\mathrm{E}_{\mathrm{c}}}} \cdot\left(0,00015-\varepsilon_{\mathrm{ct}}\right) \\
0,9 \cdot \mathrm{f}_{\mathrm{ctm}} \leq \sigma_{\mathrm{ct}} \leq \mathrm{f}_{\mathrm{ctm}}
\end{gathered}
$$

O comportamento tensão de tração vs. deformação do bambu, foi considerado elástico linear até a ruptura (Lima Jr. et al., 2000); assim, as tensões nas varas de bambu podem ser definidas com base na Eq. 14:

$$
\sigma_{\mathrm{b}}=\mathrm{E}_{\mathrm{b}} \cdot \varepsilon_{\mathrm{b}} \quad \varepsilon_{\mathrm{b}} \leq \varepsilon_{\mathrm{br}}
$$

em que $\mathrm{E}_{\mathrm{b}}$ é o módulo de elasticidade da vara de bambu, $\varepsilon_{\mathrm{b}}$ é a deformação no bambu e $\varepsilon_{\mathrm{br}}$ é a deformação de ruptura das 
varas de bambu. $O$ valor de $\varepsilon_{\mathrm{br}}$ foi determinado na primeira fase desta pesquisa, sendo de 9,04\%, com desvio-padrão de 1,02\%o (Czarnieski et al., 2004). Lima Jr. et al. (2000) mostraram que os módulos de elasticidade à tração do bambu nas regiões nodal e internodal, com os respectivos desvios-padrão, são $13 \pm$ 2,99 GPa e 23,75 $\pm 3,71 \mathrm{GPa}$, respectivamente, mas o módulo de elasticidade da armadura composta por varas de bambu com regiões nodais e internodais intercaladas, não é de fácil determinação em ensaio de tração direta; assim, o valor da referida variável foi determinado com base no ensaio experimental das oito vigas de concreto reforçadas com bambu, realizado na primeira parte do programa de pesquisa (Czarnieski et al., 2004) e ainda será discutido.

\section{Modelagem das vigas}

As dez vigas ensaiadas por Czarnieski et al. (2004) foram modeladas (Tabela 1) e discretizadas por dez elementos finitos, com dois nós e três graus de liberdade por nó (Figura 4). A área de reforço utilizada foi a área líquida da seção transversal das varas de bambu, calculada considerando-se apenas as regiões internodais. Determinou-se o módulo de elasticidade das varas de bambu, através do seguinte procedimento: a) estabeleceuse que a condição de finalização do processo de análise numérica seria a deformação limite do bambu, estabelecida igual a $9 \%$; b) para cada viga variaram-se os valores do módulo de elasticidade do bambu entre os valores de 13 e $23 \mathrm{GPa}$; c) confrontaram-se os diagramas força vs. deslocamento teóricos, com os experimentais; d) para cada viga selecionou-se o valor do módulo de elasticidade que fornecia o diagrama mais condizente com o experimental, e e) o valor adotado para o módulo de elasticidade foi a média dos oito valores obtidos. Utilizaram-se as resistências a compressão média do concreto, obtidas por ensaio de corpos-de-prova do dia do ensaio. $\mathrm{Na}$ análise foi considerada a resistência à tração do concreto, cujos valores foram calculados segundo o FIB (1999), com base na Eq. 15:

$$
\mathrm{f}_{\text {ctmin }}=0,7 \cdot 2,12 \cdot \ln \left(1+\frac{\mathrm{f}_{\mathrm{c}}}{10}\right)
$$

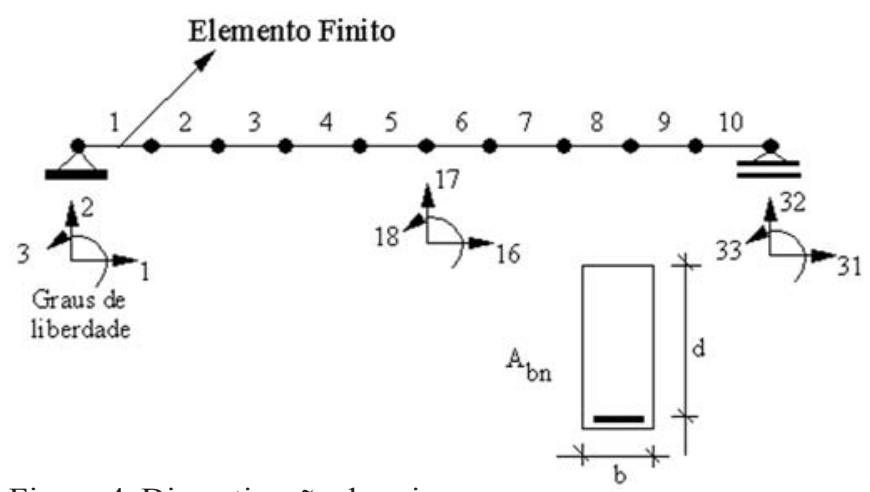

Figura 4. Discretização das vigas

\section{RESULTADOS E DISCUSSÃO}

\section{Comportamentogeral}

O módulo de elasticidade médio obtido para as varas de bambu foi de $14 \mathrm{GPa}$, significando que a resistência a tração das varas de bambu é igual a $126 \mathrm{MPa}$. Observa-se que o valor do módulo de elasticidade das varas de bambu ficou bastante próximo ao do módulo de elasticidade da região nodal e a resistência à tração foi $30 \%$ superior à resistência da região nodal, cujo valor é $97 \mathrm{MPa}$ (Lima Jr. et al., 2000).

Nas Figuras 5 e 6 apresentam-se as curvas teóricas e experimentais força vs. deslocamento e momento vs. deformação do bambu, das dez vigas ensaiadas por Czarnieski et al. (2004). Nota-se que o modelo computacional utilizado representou, com boa aproximação, o comportamento das vigas de concreto reforçadas com bambu, seja no que se refere aos deslocamentos ou às deformações. A diferença entre as forças últimas teóricas e experimentais das vigas de concreto armadas com aço, foi de apenas $5 \%$; já para as vigas armadas com bambu foi de $3 \%$; deste modo, conclui-se que a precisão do modelo computacional em predizer a capacidade resistente das vigas armadas com bambu é similar à precisão obtidas para as vigas armadas com aço. Vê-se, em todos os diagramas força vs. deslocamento teóricos, um trecho serrilhado entre o deslocamento nulo e $5 \mathrm{~mm}$, cujas oscilações são provenientes

Tabela 1. Características das vigas

\begin{tabular}{|c|c|c|c|c|c|c|c|c|c|}
\hline Espécime & Tipo de armadura & $A_{b}\left(\mathrm{~cm}^{2}\right)$ & $A_{b n}\left(\mathrm{~cm}^{2}\right)$ & $\mathrm{f}_{\mathrm{cj} 14}-\mathrm{s}(\mathrm{MPa})$ & $\mathrm{d}(\mathrm{cm})$ & $\mathrm{L}(\mathrm{cm})$ & $\mathrm{h}(\mathrm{cm})$ & $\mathrm{b}(\mathrm{cm})$ & $2 \mathrm{P}(\mathrm{kN})$ \\
\hline Viga 1 & Aço - $2 \phi 8,0$ & 1,00 & 1,00 & $27,16-0,75$ & 23,8 & \multirow{10}{*}{250} & \multirow{10}{*}{25} & \multirow{10}{*}{10} & 41,20 \\
\hline Viga 2 & Aço - $2 \phi 8,0$ & 1,00 & 1,00 & $27,11-0,20$ & 23,9 & & & & 39,83 \\
\hline Viga 3 & Bambu $-2 \phi(2 \times 1)$ & 5,04 & $? 4,50$ & $27,03-1,08$ & 23,6 & & & & 33,04 \\
\hline Viga 4 & Bambu $-2 \phi(2 \times 1)$ & 5,21 & $? 4,50$ & $26,26-0,48$ & 23,7 & & & & 32,90 \\
\hline Viga 5 & Bambu $-4 \phi(1 \times 1)$ & 4,75 & $? 4,50$ & $29,46-1,11$ & 23,7 & & & & 26,27 \\
\hline Viga 6 & Bambu $-4 \phi(1 \times 1)$ & 5,03 & $? 4,50$ & $28,97-1,13$ & 23,7 & & & & 28,66 \\
\hline Viga 7 & Bambu - $4 \phi(2 \times 1)$ & 8,76 & $? 9,00$ & $28,77-1,40$ & 22,3 & & & & 44,62 \\
\hline Viga 8 & Bambu - $4 \phi(2 \times 1)$ & 9,42 & $? 9,00$ & $28,56-1,67$ & 22,6 & & & & 58,53 \\
\hline Viga 9 & Bambu $-8 \phi(1 \times 1)$ & 12,11 & $? 9,00$ & $26,69-1,78$ & 22,5 & & & & 57,16 \\
\hline Viga 10 & Bambu $-8 \phi(1 \times 1)$ & 11,40 & $? 9,00$ & $27,73-0,80$ & 22,3 & & & & 58,06 \\
\hline
\end{tabular}


Vigas de concreto reforçadas com bambu Dendrocalamus giganteus. II: Modelagem e critérios

657

A.

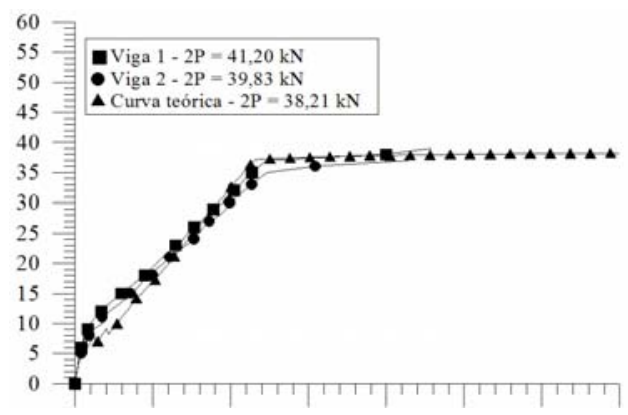

B.

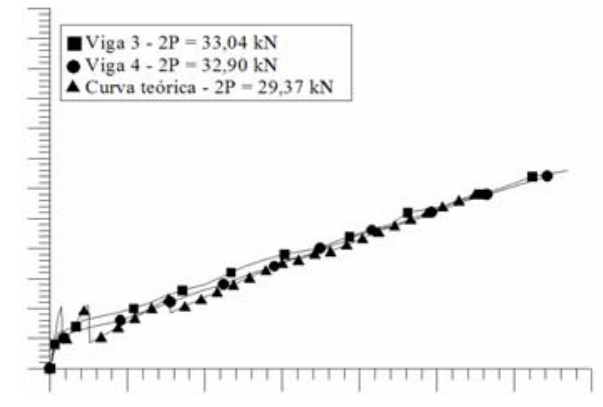

C.

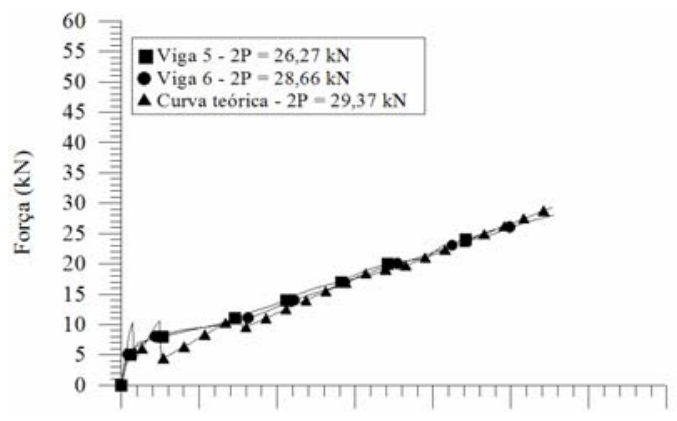

D.

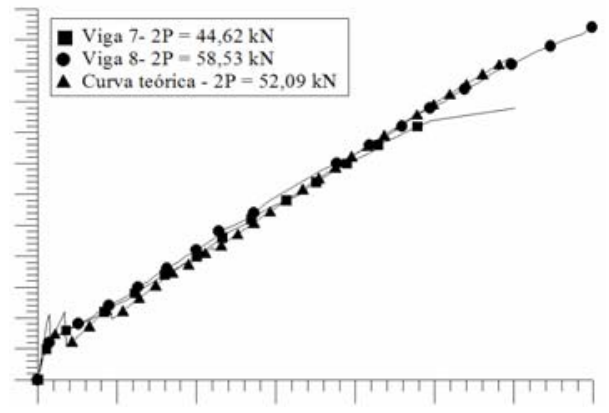

E.

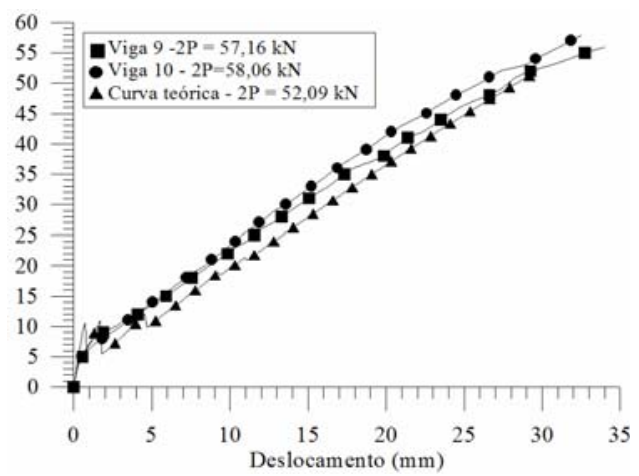

Figura 5. Curvas teóricas e experimentais força vs. deslocamento das vigas ensaiadas por Czarnieski et al. (2004):

A. vigas 1 e 2; B. vigas 3 e 4; C. vigas 5 e 6 ; D. vigas 7 e 8 , e

E. vigas 9 e 10
A.

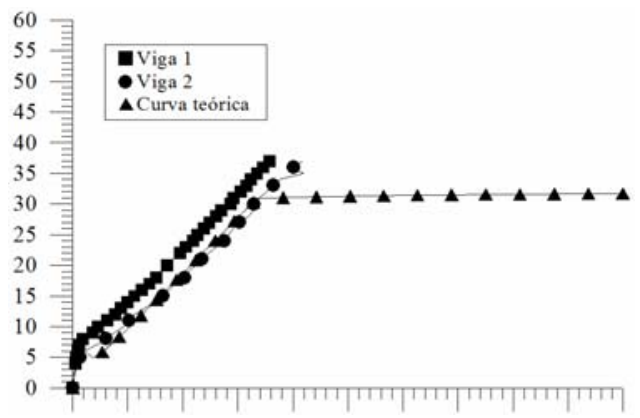

B.

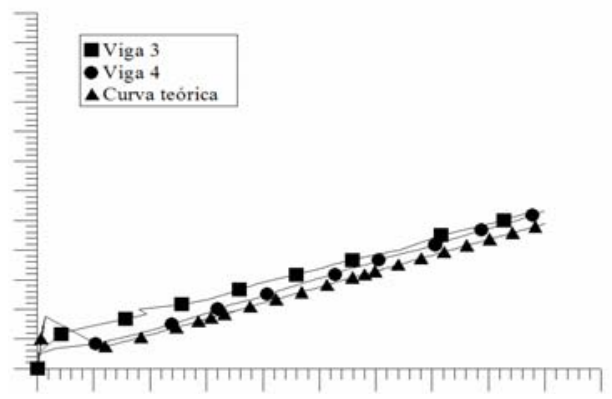

C.

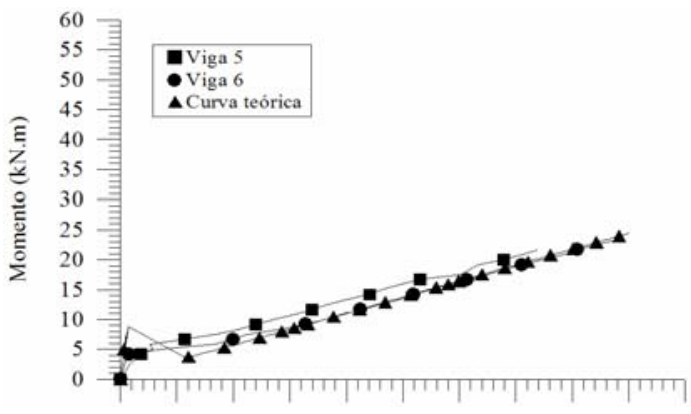

D.

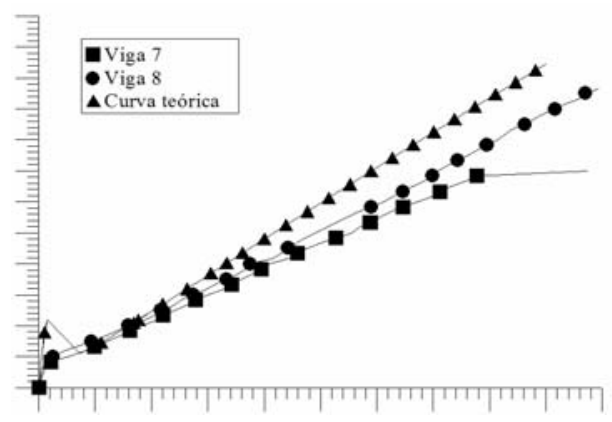

E.

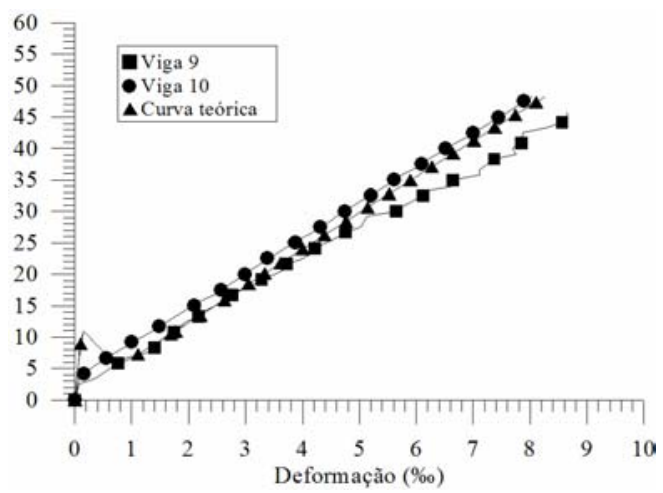

Figura 6. Curvas teóricas e experimentais momento vs. deformação das vigas ensaiadas por Czarnieski et al. (2004): A. vigas 1 e 2; B. vigas 3 e 4; C. vigas 5 e 6; D. vigas 7 e 8 , e E. vigas 9 e 10

R. Bras. Eng. Agríc. Ambiental, Campina Grande, v.9, n.4, p.652-659, 2005 
da fissuração do concreto das vigas, em que o primeiro dente corresponde ao aparecimento da primeira fissura, o segundo ao da segunda fissura, e assim sucessivamente. A força de fissuração teórica foi bem superior à experimental, fato este decorrente da superestimativa da resistência à tração do concreto da Eq. 16 sugerida pelo FIB (1999). Finalmente, notase que as vigas de concreto reforçadas com bambu podem ser modeladas pelos programas usuais de concreto armado, considerando-se um diagrama elástico linear, até a ruptura para o bambu. Este fato re-convalida as afirmações de Czarnieski et al. (2004) que mostraram que o comportamento das vigas de concreto reforçadas com bambu segue a teoria usual de flexão adotada para o concreto armado com barras de aço.

\section{Critérios de dimensionamento}

Para o dimensionamento de vigas de concreto reforçadas com bambu, as hipóteses e sugestões seguintes podem ser adotadas:

1. até a ruptura, as seções transversais permanecem planas e perpendiculares à linha neutra;

2. o encurtamento de ruptura do concreto é de $3,5 \%$ e a tensão máxima de compressão deve ser de 0,85 .fcd;

3. considerando-se, com base nos ensaios de tração realizados por Lima Jr. et al. (2000), um desvio padrão da ordem de $15 \mathrm{MPa}$ observado para a região nodal; ter-se-á o valor da resistência à tração das varas de bambu, obtido no presente trabalho e, ainda, que tal resistência segue uma distribuição Normal e que apenas $5 \%$ dos valores da resistência sejam inferiores ao valor característico, sugere-se que a resistência característica do bambu Dendrocalamus giganteus, fbk, seja tomada igual a $105 \mathrm{MPa}$ e o módulo de elasticidade igual a $90 \%$ de $14 \mathrm{MPa}$. O coeficiente de minoração da resistência característica pode ser tomado igual a 1,8 ;

4. é totalmente desprezada a resistência à tração do concreto e não se sugere a utilização de concretos com resistências características superiores a $25 \mathrm{MPa}$, por questões econômicas;

5. não se deve utilizar taxas de armadura longitudinal inferiores a $0,9 \%$.bw.d, sendo bw a largura da seção transversal e d a altura útil da viga;

6. não é aconselhável a utilização de armadura composta por varas de bambu para vãos superiores a 3,5 m;

7. enfim, os demais critérios de dimensionamento devem seguir os procedimentos usuais do concreto armado, sugeridos pela NBR6118:2003 (ABNT, 2003).

Com o objetivo de exemplificar os critérios sugeridos, dimensionou-se uma viga de concreto armado que, em seguida, foi analisado por meio do método dos elementos finitos. Os detalhes do dimensionamento e da modelagem são apresentados no exemplo a seguir.

\section{Exemplo}

Seja uma viga de concreto com resistência característica à compressão de $20 \mathrm{MPa}$ reforçada com bambu, vão de centro a centro de apoio de $3,5 \mathrm{~m}$ e seção transversal de $15 \mathrm{~cm}$ por $40 \mathrm{~cm}$; esta viga faz parte da estrutura de uma construção rural e serve de suporte para a laje do primeiro pavimento, que possui 3,5 m de vão livre. Determinar a área necessária de bambu Dendrocalamus giganteus.

$1^{\circ}$ passo: Carga na viga:

A carga na viga será o somatório da parcela do carregamento oriundo da laje mais a parcela referente ao peso da parede e da viga; assim, ter-se-á:

$$
\begin{aligned}
& \text { Peso próprio }=0,15 \cdot 0,40 \cdot 25,0=1,5 \mathrm{kN} \mathrm{m}^{-1} \\
& \text { Carga parede }=0.15 \cdot 2 \cdot 12,0=3,60 \mathrm{kN} \mathrm{m}^{-1} \\
& \text { Carga laje }=(1,50+0,50+2,50) \cdot 3,5 / 2=7,88 \mathrm{kN} \mathrm{m}^{-1} \\
& \text { Carga viga }=12,98 \mathrm{kN} \mathrm{m}^{-1}
\end{aligned}
$$

$2^{\circ}$ passo: Momento máximo atuante de cálculo:

$$
\mathrm{M}_{\mathrm{d}}=\frac{1.4 \cdot \mathrm{q} \cdot \mathrm{L}^{2}}{8}=\frac{1.4 \cdot 12,98 \cdot 3,5^{2}}{8} \cong 27,83 \mathrm{kN} \mathrm{m}
$$

$3^{\circ}$ passo: Cálculo da armadura de bambu com base na Figura 7 :
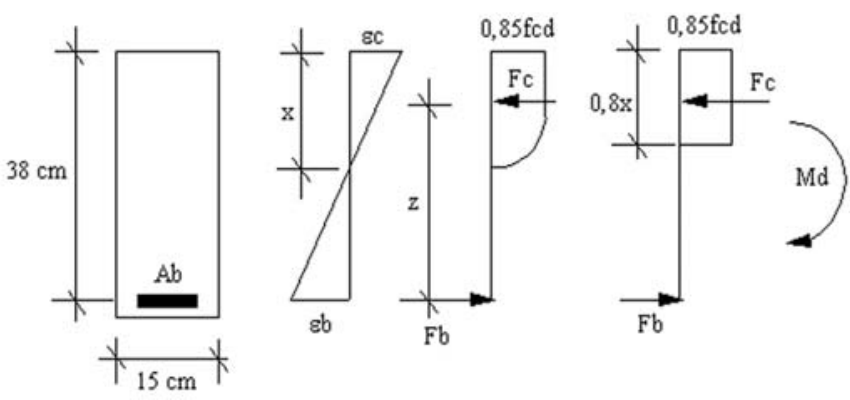

Figura 7. Equilíbrio da seção transversal

$$
\begin{gathered}
\sum M b=0=>M d=0,85 \cdot f_{c d} \cdot 0,8 \cdot x \cdot b_{w} \cdot(d-0,4 \cdot x) \\
=>x=5,32 \mathrm{~cm} \\
\sum F_{x}=0 \Rightarrow F_{c}=F_{b}=>0,85 \cdot f_{c d} \cdot 0,8 \cdot x \cdot b_{w}= \\
A_{b} \cdot \frac{f_{b k}}{1,8}=>A_{b}=13,29 \mathrm{~cm}^{2}
\end{gathered}
$$

$\mathrm{Ab}=>7$ varas de $2 \mathrm{~cm} \times 1 \mathrm{~cm}=2,45 \% \cdot \mathrm{b}_{\mathrm{w}} \cdot \mathrm{d}$ ok! $4^{\circ}$ passo: Flecha máxima permitida:

$\mathrm{f}($ carga permanente $+70 \%$ da carga acidental $) \leq \frac{350}{300}=1,17 \mathrm{~cm}$

$$
\mathrm{f}_{\text {teórica }} \approx 1,20 \mathrm{~cm}
$$

Para verificar o comportamento estrutural da viga dimensionada, utilizou-se o programa descrito no presente 


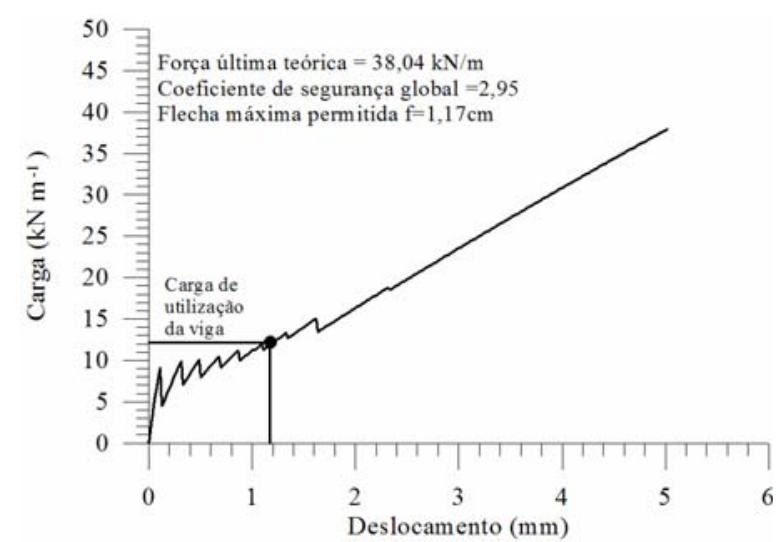

Figura 8. Curva carga vs. deslocamento da viga dimensionada no exemplo

trabalho para a obtenção do diagrama força vs. deslocamento da viga. Na Figura 8, apresenta-se o diagrama força vs. deslocamento da viga dimensionada e se observa que o coeficiente global de segurança da viga foi de 2,95, valor este equivalente aos encontrados nas vigas de concreto armadas com aço, que variam de 2,5 a 3,5, significando que a viga de bambu trabalhará com uma carga de cerca de $33 \%$ de sua resistência. Por outro lado, a viga mostrou-se bastante flexível, apresentando flecha para a carga de serviço $3 \%$ maior que a máxima permitida, fato que se deve ao baixo módulo de elasticidade do bambu.

\section{CONCLUSÕES}

1. O modelo computacional proposto foi capaz de representar, com boa acuracidade, o comportamento estrutural das vigas de concreto reforçadas com bambu, tanto no que diz respeito aos deslocamentos e deformações como às forças últimas.

2. As vigas de concreto reforçadas com bambu obedecem à teoria de Bernoulli-Kirchoff, sendo possível a aplicação dos procedimentos usuais de dimensionamento das estruturas de concreto armado, considerando-se apenas o diagrama tensão vs. deformação do reforço elástico-linear.

3. O módulo de elasticidade global das varas de bambu é de $14 \mathrm{GPa}$ e a resistência a tração de $126 \mathrm{MPa}$.

4. Os critérios sugeridos para o dimensionamento das vigas de concreto reforçadas com bambu se mostraram adequados; contudo, é necessário um estudo mais abrangente sobre os limites de deslocamento e magnitude das flechas admissíveis, para esses elementos estruturais.

\section{AGRADECIMENTOS}

Os autores são gratos ao Professor Normando Perazzo Barbosa, da Universidade Federal da Paraíba, pelas sugestões.

\section{LITERATURA CITADA}

ABNT - Associação Brasileira de Normas Técnicas. NBR6118:2003 - Projeto de estruturas de concreto: Procedimentos. Rio de Janeiro, 2003, 232p.
Beraldo, A.L. Bambucreto - o uso do bambu como reforço do concreto. In: Congresso Brasileiro de Engenharia Agrícola, 16, 1987, Jundiaí. Anais... Jundiaí, SP: SBEA, v.2, 1987, p.521530.

Campos, C.M.O. Um modelo computacional para análise de vigas de concreto protendido com cabos aderentes e não aderentes. Rio de Janeiro: PUC, 1993. 83p. Dissertação Mestrado

CEB. Fip model code 1990 - Final draft., London, Comité Euro International du Béton, 1990.460p. Bulletin d'Information

Cordero, V.M. Casa de Painéis de bambu-argamassa. São Carlos: USP, 1990. 147p. Dissertação Mestrado

Culzoni, R.A.M. Características dos bambus e sua utilização como material alternativo no concreto. Rio de Janeiro: PUC, 1985. 134p. Dissertação Mestrado

Czarnieski, C.J.; Mesquita, L.P.; Braga Filho, A.C; Willrich, F.L.; Lima Jr., H.C.; Barbosa, N.P. Avaliação da aderência bambuconcreto. In: Jornadas Sud-Americanas de Ingeniería Estructural, 31, 2004, Anais.... Mendoza, 2004, CD-rom

Ferrão, A.M.; Freire, W.J. Aderência entre bambu e concreto: teste de arrancamento com taliscas de Bambusa tuldoides. In: Encontro Brasileiro em Madeiras e em Estruturas de Madeira, 5, 1995, Belo Horizonte. Anais... Belo Horizonte: EBRAMEM, v.2, 1995, p.389-398.

Ferreira, G.C.S.; Moreno Jr., A.L; Beraldo, A.L. Reinforced concrete bamboo beam behavior. In: Agribuilding 2001. Proceedings... Campinas, 2001, p.331-337.

FIB. Structural concrete - Textbook on behavior, design and performance. Updated knowledge of the CEB/FIP Model Code 1990. Comité Euro International du Béton, London, v.1, 1999, 244p.

Ghavami, K; Hombeck, R.V. Mechanical properties and waterrepellent treatment of bamboo. In: Latin American Symposium Rational Organization of Building Applied to Low Cost Housing. 1981, São Paulo. Proceedings... São Paulo, v.1, 1981, p.49-55.

Krüger, S.D. Uma metodologia para a análise de pórticos planos de concreto armado sujeitos a grandes deslocamentos. Rio de Janeiro: PUC, 1989. 180 p. Dissertação Mestrado

Lima Jr., H.C.; Dalcanal, P.R.; Willrich, F.L.; Barbosa, N.P. Características mecânicas do bambu Dendrocalamus giganteus: análise teórica e experimental. In: Barbosa, N.P.; Swamy, R.N. and Lynsdale, C. (eds.). Sustainable construction into the next millennium: Environmentally friendly and innovative cement based materials. João Pessoa:SNT, 2000, p.394-406.

Lima Jr., H.C; Xavier, A.C.; Barbosa, N.P.; Toledo Filho, R.D. Vigas de concreto reforçadas com bambu. In: Congresso de Engenharia Civil da Universidade Federal de Juiz de Fora, 1, 1994, Anais... Juiz de Fora, v.1, 1994, p.22-32.

Lima Jr., H.C.; Xavier, A.C.; Barbosa, N.P.; Toledo Filho, R.D. Aderência bambu-concreto. In: Congresso de Engenharia Civil da Universidade Federal de Juiz de Fora, 2, 1996, Anais... Juiz de Fora. v.1, 1996, p.312-323.

Lopez, H.O. Bambú: su cultivo y aplicaciones en: Fabricación de papel, construcción, arquitectura, ingeniería, artesanía. Cali: SNT, 1974, 318p. Boletín Técnico

Rosa, S.P.A.P. da. Análise teórica e experimental de colunas de concreto armado com bambu. Rio de Janeiro: PUC, 2002. 135p. Dissertação Mestrado 